\title{
Evaluation of Erectile Function in Men with Lower Urinary System Symptoms
}

(D) Murat Dursun 1 , (D) Hüseyin Beşiroğlu²

${ }^{1}$ Malatya Training and Research Hospital, Department of Urology, Malatya, Turkey

2istanbul Çatalca ilyas Çokay State Hospital, Urology, istanbul, Turkey

\section{Abstract}

Objective: The aim of this study was to investigate the possible relationship between erectile dysfunction (ED) and lower urinary tract symptoms (LUTS).

Methods: Sixty-two patients with LUTS due to benign prostatic hyperplasia (BPH) were included in the study. Fifty-eight healthy male patients were included in the study as control group. Ages, comorbidities, body mass index and previous surgeries of the patients were determined. The patients were asked to complete the International Prostate Symptom Score and the International Index of Erectile Function (IIEF-5) questionnaires.

Results: The mean age was 61.41 (41-78) years in the patients with LUTS and 62.34 (40-81) years in the control group. There was no statistically significant difference between the two groups in terms of age. It was observed that LUTS severity increased significantly with age ( $p<0.05)$. When all subjects were evaluated, there was a statistically significant relationship between LUTS and ED. When the IIEF- 5 score less than 22 is accepted as ED, ED was present in $59.06 \%$ of patients with LUTS and $29.1 \%$ in asymptomatic patients. In addition, the rate of ED was significantly increased with age, as expected $(p<0.05)$.

Conclusion: The main finding in our study was that LUTS was independently associated with ED. Because of the relationship between LUTS/BPH and male sexual dysfunction, patients presenting with any of these conditions should be routinely screened for other conditions. A better understanding of the molecular pathways beneath this relationship can help to better define clinical trials.

Keywords: Benign prostatic hyperplasia, erectile dysfunction, IIEF, International Prostate Symptom score, lower urinary tract symptoms

\section{INTRODUCTION}

The prevalence of sexual problems in the general population has increased in recent years and erectile dysfunction (ED) is one of the most common types of sexual dysfunction in men worldwide (1, 2). Studies have shown that sexual functions and ED are important factors on quality of life (3-5). ED can affect functions in all aspects of life, including emotional, social, sexual, recreational and intellectual areas (5).

In recent years, studies showing the relationship between lower urinary tract symptoms (LUTS) and ED are noteworthy. Although the mechanisms underlying the association between LUTS and ED in men with benign prostatic hyperplasia (BPH) have not been fully elucidated, various pathophysiological theories have been proposed in the literature and the possible linkages between these pathways are still being investigated (6). The pathophysiology of LUTS and its underlying mechanisms is not yet fully understood (7), however, the presence of concomitant ED or vice versa in many patients with LUTS suggests that its pathophysiological mechanisms may be similar to ED (8). Men with LUTS have one or more physiological diagnoses, some comorbidities and/or risk factors. Nearly $70 \%$ of men with LUTS/BPH have ED. Recent treatment trends for LUTS/BPH include the use of various pharmacological agents, such as

Cite this article as:Dursun M, Beșiroğlu H. Evaluation of Erectile Function in Men with Lower Urinary System Symptoms. Eur Arch Med Res 2019; 35 (1):23-26

๑Copyright 2019 by the Health Sciences University, Okmeydanı Training and Research Hospital

European Archives of Medical Research published by Galenos Publishing House. 
tadalafil, a phosphodiesterase-5 (PDE-5) inhibitors (9). Recent epidemiological studies have shown that there is a relationship between LUTS/BPH and ED independent of comorbidities such as age and hypertension, diabetes, dyslipidemia and coronary heart disease $(10,11)$. In contrast, in some other studies, no association was found between LUTS and ED (12-14). On the other hand, the medical or surgical treatments in patients with LUTS/BPH can significantly affect erectile functions, or similarly, PDE inhibitors used for ED treatment may also improve LUTS (15, 16). Because of these relationships, we aimed to evaluate the possible relationship between LUTS and ED in patients admitted to urology outpatient clinic with LUTS.

\section{METHODS}

Sixty-two male patients who applied to urology outpatient clinic due to BPH-related LUTS were included in the study as patient group. Fifty-eight healthy men who admitted for other reasons were included in the study as control group. Ages, comorbidities, body mass index (BMI) and previous surgeries of the patients were determined. Smokers, alcohol and drug addicts, patients with previous pelvic or lumbar surgery/trauma, patients with Peyronie's disease and antidepressant drug use were excluded from the study. In addition, those who were treated for LUTS or prostate disease within the last 6 months were excluded from the study. Informed consent form was obtained from all participants. Institutional review board approval was obtained from the local ethical committee.

The patients were asked to complete the International Prostate Symptom Score (IPSS) and the International Index of Erectile Function (IIEF-5) questionnaires. LUTS was rated according to the IPSS scoring system (0: normal, symptom score less than of equal to 7: mild, symptom score range 8-19: moderate, symptom score range 20-35: severe). ED was classified into five categories based on the scores as suggested by Rosen et al. (11): no ED (IIEF-5 score 22-25), mild (IIEF-5 score 17-21), moderate (IIEF-5 score 8-16) and severe (IIEF-5 score $<8$ ).

\section{Statistical Analysis}

Data were analyzed using SPSS 14.0. Chi-square and Student's t-test were used to compare the data. P value less than 0.05 was considered statistically significant.

\section{RESULTS}

A total of 120 men between the ages of 40 and 81 were included in the study. The study group consisted of 62 patients who were admitted with LUTS (patient group) and 58 patients who applied for other reasons (control group). The mean age was 61.41 (41-78) years in the patients with LUTS and 62.34 (40-81) years in the control group. There was no statistically significant difference between the two groups in terms of age. When BMI was evaluated, the mean BMI was found to be $29.11 \pm 2.87$ in the patient group and $27.27 \pm 2.97$ in the control group. BMI was significantly higher in men with LUTS than those without LUTS $(p<0.05)$. Regarding IPSS scores, the mean total IPSS score was 18.2 (5-35) in patients with LUTS. Of 62 patients, 18 had mild, 24 had moderate and 20 had severe symptoms. It was observed that LUTS severity increased significantly with age $(p<0.05)$.

When all subjects were evaluated, there was a statistically significant relationship between LUTS and ED. When the IIEF-5 score less than 22 is accepted as ED, ED was present in 59.6\% of patients with LUTS and $29.1 \%$ in asymptomatic patients. When IIEF-5 scores were compared between the two groups, IIEF-5 scores were found to be significantly lower in the patient group. The mean IIEF-5 was $15.8 \pm 1.38$ in the patient group and $21.8 \pm 1.56$ in the control group. IIEF-5 scores were negatively correlated with the severity of LUTS $(p<0.05)$. In addition, the rate of ED was significantly increased with age, as expected $(p<0.05)$. All results are summarized in Tables 1 and 2 .

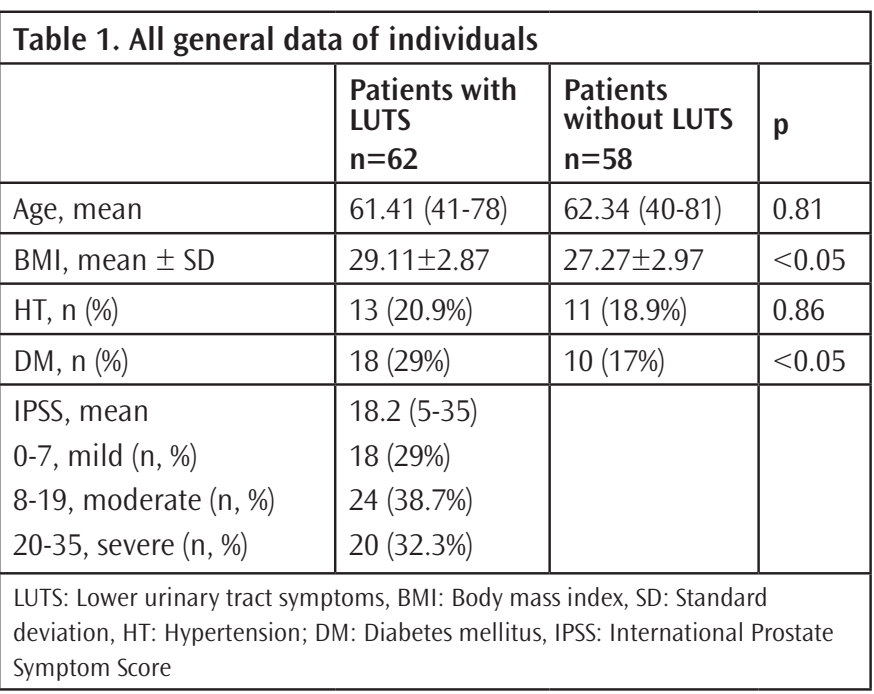

Table 2. Comparison of erectile dysfunction rates in the patient and control groups

\begin{tabular}{|l|l|l|l|}
\hline & $\begin{array}{l}\text { Patients with } \\
\text { LUTS } \\
\mathbf{n}=62\end{array}$ & $\begin{array}{l}\text { Patients without } \\
\text { LUTS } \\
\mathbf{n}=58\end{array}$ & $\mathbf{p}$ \\
\hline ED, $\mathrm{n}(\%)$ & $37(59.6)$ & $17(29.3)$ & $<0.05$ \\
\hline IIEF, mean \pm SD & $15.8 \pm 1.38$ & $21.8 \pm 1.56$ & $<0.05$ \\
\hline
\end{tabular}

LUTS: Lower urinary tract symptoms, ED: Erectile dysfunction, IIEF: International Index of Erectile Function 


\section{DISCUSSION}

Sexuality is an indispensable factor in quality of life, thus, the sexual life of elderly individuals has prolonged with the emergence of oral therapies developed for ED. ED is a complex situation with risk factors such as old age, comorbid conditions, some drugs, obesity, lifestyle behaviors, alcohol and tobacco use. As a result of this complex situation, the relationship between ED and LUTS has not been fully elucidated. There are strong epidemiological findings showing a significant relationship between LUTS and sexual dysfunction in elderly men in the world, regardless of age and other comorbidities (17-19).

In this study, we also evaluated the relationship between LUTS and ED. The main finding of our study was that LUTS was independently associated with ED. We have tried to determine the prevalence and predictors of ED in patients with and without LUTS. In this study, the prevalence of ED was significantly higher in patients with LUTS than those without LUTS ( $58.4 \%$ vs. $29.1 \%$, respectively). The high ED prevalence we found in LUTS is similar to that of the Cologne male survey study with 8000 individuals in Germany. In this study, ED prevalence was $72 \%$ in men with LUTS (20). Moreover, the higher prevalence in this study suggests that the majority of men with LUTS in this country have ED findings, but that they do not seek medical advice. It is known that the severity of ED and LUTS increases with age. There are several studies showing this finding and our study is consistent with these studies $(21,22)$. The multinational survey of the aging male study conducted with approximately 14.000 men in the United States and six European countries has showed that ED is strongly associated with LUTS (23). This study reported that most men over 50 years of age were sexually active, but the number of sexual intercourse decreased with increased LUTS severity (up to 7.5 per month for men in their 60 s, up to 3.2 per month for men aged 80 years). ED was reported by approximately $49 \%$ of the study cohort and the IIEF score was strongly correlated with LUTS severity.

The relationship between LUTS/BPH and male sexual dysfunction has been explained by various pathophysiological mechanisms. These include autonomic hyperactivity, changes in the Rho/Rho kinase pathway, microvascular dysfunction, endothelial [nitric oxide (NO) synthase/NO] dysfunction, pelvic ischemia and agerelated hormone imbalances (21). The role of NO in the prostate and penis has been extensively studied, particularly following the development of PDE-5 inhibitors, the first-line treatment for ED. The role of NO in erection is known. NO has been shown to be present in prostate tissue and be effective in smooth muscle tone (24). On the other hand, although PDE5-inhibitors showed significant improvement in both LUTS and ED in men with $\mathrm{BPH}$, these treatments did not affect the urinary flow rate. Therefore, the mechanisms of action of these drugs on LUTS are not fully understood. On the other hand, metabolic syndrome and its components have been shown to be associated with the development of LUTS and ED in various clinical trials $(25,26)$. This is considered to be one of the reasons for the common pathophysiology and strong relationship. In addition, insulin resistance and developing secondary hyperinsulinemia have been shown to play a role in the pathogenesis of LUTS as well as ED in these studies.

Because of the strong association between LUTS/BPH and male sexual dysfunction, patients presenting with any of these conditions should be routinely screened and questioned for the other disease. In addition, patients with LUTS/BPH should be monitored for treatment-related sexual consequences, as sexual side effects may occur in patients undergoing medical and surgical treatment for LUTS/BPH $(16,21,27,28)$. Beyond the IPSS and IIEF evaluation, it is important to evaluate additional diseases such as the metabolic syndrome and the use of drugs associated with these diseases. It is also important to give advice on lifestyle changes in patients with both disorders (29). Sexual functions should always be evaluated before starting LUTS/BPH treatment. If sexual dysfunction is detected, lifestyle changes such as weight loss and increasing physical activity should be planned. Given the need for medication or surgical treatment for LUTS/BPH, the possible effects of these therapies on ED should be discussed with patients.

\section{CONCLUSION}

In conclusion, LUTS and ED are age-related disorders that are quite common. Although no coincidental relationship was found, various epidemiological studies and potential common biological mechanisms confirm the existence of this relationship. Considering that many elderly men do not ask for help with their sexual problems and physicians do not often question their sexual life, it is recommended that men presenting with LUTS should be evaluated for sexual dysfunction and ED. Similarly, patients presenting with ED should also be evaluated for LUTS. In clinical practice, it should be noted that the presence of concomitant ED in the management of LUTS and some of the current medical treatments of LUTS/BPH may have positive or negative effects on sexual functions. A better understanding of the molecular pathways behind this relationship as a result of clinical trials can help to identify new possible targets and develop new treatment approaches to manage both disorders.

\section{Ethics}

Ethics Committee Approval: The study was approved by the Malatya Clinical Research Ethics Committee (Approval no: 2018/24). 
Informed Consent: Informed consent form was obtained from all participants.

Peer-review: Externally peer-reviewed.

\section{Authorship Contributions}

Concept: M.D., Design: M.D., Data Collection or Processing: M.D., H.B., Analysis or Interpretation: H.B., Literature Search: M.D., H.B., Writing: M.D.

Conflict of Interest: No conflict of interest was declared by the authors.

Financial Disclosure: The authors declared that this study received no financial support.

\section{REFERENCES}

1. Shaeer KZ, Osegbe DN, Siddiqui SH, Razzaque A, Glasser DB, Jaguste V. Prevalence of erectile dysfunction and its correlates among men attending primary care clinics in three countries: Pakistan, Egypt, and Nigeria. Int J Impot Res 2003;1:S8-14

2. Eardley I. The incidence, prevalence, and natural history of erectile dysfunction. Sex Med Rev 2013;1:3-16.

3. Oladiji F, Kayode 00, Parakoyi DB. Influence of socio- demographic characteristics on prevalence of erectile dys- function in Nigeria. Int J Impot Res 2013;25:18-23.

4. Braun M, Wassmer G, Klotz T, Reifenrath B, Mathers M, Engelmann U. Epidemiology of erectile dysfunction: results of the 'Cologne Male Survey'. Int J Impot Res 2000;12:305-11.

5. Rosen R, Altwein J, Boyle P, Kirby RS, Lukacs B, Meuleman E, et al. Lower urinary tract symptoms and male sexual dys- function: the multinational survey of the aging male (MSAM-7). Eur Urol 2003;44:637-49.

6. McVary KT. Erectile dysfunction and lower urinary tract symp- toms secondary to BPH. Eur Urol 2005;47:838-45.

7. Andersson KE, de Groat WC, McVary KT, Lue TF, Maggi M, Roehrborn CG, et al. Tadalafil for the treatment of lower urinary tract symptoms secondary to benign prostatic hyper- plasia: pathophysiology and mechanism(s) of action. Neurourol Urodyn 2011;30:292-301.

8. Kirby M, Chapple C, Jackson G, Eardley I, Edwards D, Hackett G, et al. Erectile dysfunction and lower urinary tract symptoms: a consensus on the importance of co-diagnosis. Int J Clin Pract 2013;67:606-18.

9. Oelke M, Bachmann A, Descazeaud A, Emberton M, Gravas S, Michel MC, et al. EAU guidelines on the treat- ment and follow-up of non-neurogenic male lower urinary tract symptoms including benign prostatic obstruction. Eur Urol 2013;64:118-40.

10. Schiff JD, Mulhall JP. The link between LUTS and ED: clinical and basic science evi- dence. J Androl 2004;25:470-8.

11. Rosen RC. Update on the relationship between sexual dysfunction and lower urinary tract symptoms/benign prostatic hyperplasia. Curr Opin Urol 2006;16:11-19.

12. Huang WJ, Chen KK, Chang LS. Correlation between voiding and erectile function in patients with symptomatic benign prostatic hyperplasia. J Chin Med Assoc 2005;68:178-82.
13. Leungwattanakij S, Roongreungsilp U, Lert- sithichai P, Ratana-Olarn K. The association between erectile function and severity of lower urinary tract symptoms. J Med Assoc Thai 2005;88:91-5.

14. Marionneau N, Perrin P, Taieb C. Lower uri-12 nary tract symptoms are poorly correlated with erectile dysfunction (in French). Prog Urol 2006;16:572-7.

15. Roehrborn CG. Lower urinary tract symp- 15 toms, benign prostatic hyperplasia, erectile dysfunction, and phosphodiesterase-5 in- hibitors. Rev Urol 2004;6:121-7.

16. Kaplan SA, Gonzalez RR, Te AE. Combination of alfuzosin and sildenafil is superior to monotherapy in treating lower urinary tract symptoms and erectile dysfunction. Eur Urol 2007;51:1717-23.

17. Gacci M, Eardley I, Giuliano F, Hatzichristou D, Kaplan SA, Maggi M, et al. Critical Analysis of the Relationship Between Sexual Dysfunctions and Lower Urinary Tract Symptoms Due to Benign Prostatic Hyperplasia. Eur Urol 2011;60:809-25.

18. Ikuerowo SO, Akindiji YO, Akinoso OA, Akinlusi FM, Esho JO. Association between Erectile Dysfunction and Lower Urinary Tract Symptoms due to Benign Prostatic Hyperplasia in Nigerian Men. Urol Int 2008 ;80: 296-9.

19. De Nunzio C, Roehrborn CG, Andersson KE, McVary KT. Erectile Dysfunction and Lower Urinary Tract Symptoms. Eur Urol Focus 2017;3:352-63.

20. Braun M, Wassmer G, Klotz T, Reifenrath B, Mathers M, Engelmann U. Epidemiology of erectile dysfunction: results of the 'Cologne Male Survey'. Int J Impot Res 2000; 12: 305- 11.

21. Schiff JD, Mulhall JP. The link between LUTS and ED: clinical and basic science evi- dence. J Androl 2004;25:470-8.

22. Stroberg P, Boman H, Gellerstedt M, Hedelin H. Relationships between lowe urinary tract symptoms, the bother they induce and erectile dysfunction. Scand J Urol Nephrol 2006;40:307-12.

23. Rosen R, Altwein J, Boyle P, Kirby RS, Lukacs B, Meuleman E, et al. Lowe urinary tract symptoms and male sexual dys- function: the multinational survey of the aging male (MSAM-7). Eur Urol 2003;44:637-49.

24. Gacci M, Andersson K-E, Chapple C, Maggi M, Mirone V, Oelke M, et al. Latest evidence on the use of phosphodiesterase type 5 inhibitors for the treatment of lower urinary tract symptoms secondary to benign prostatic hyperplasia. Eur Urol 2016;70:124-33.

25. De Nunzio C, Cindolo L, Gacci M, Pellegrini F, Carini M, Lombardo R, et al Metabolic syndrome and lower urinary tract symptoms in patients with benign prostatic enlargement: a possible link to storage symptoms. Urology 2014;84:1181-7.

26. McVary K. Lower urinary tract symptoms and sexual dysfunction: epidemiology and pathophysiology. BJU Int 2006;97:23-8.

27. Roehrborn CG. Lower urinary tract symp- 15 toms, benign prostatic hyperplasia, erectile dysfunction, and phosphodiesterase-5 in- hibitors. Rev Urol 2004:6:121-7.

28. McVary KT, Monnig W, Camps JL Jr, Young JM, Tseng LJ, van den Ende G. Sildenafil ci- trate improves erectile function and urinary symptoms in men with erectile dysfunction and lower urinary tract symptoms associat- ed with benign prostatic hyperplasia: a ran- domized, double-blind trial. J Urol 2007;177:1071-7.

29. Fusco F, D’Anzeo G, Sessa A, Pace G, Rossi A, Capece M, et al. BPH/LUTS and ED: common pharmacological pathways for a common treatment. J Sex Med 2013;10:2382-93. 